\title{
miR-7-5p inhibits cell migration and invasion in glioblastoma through targeting SATB1
}

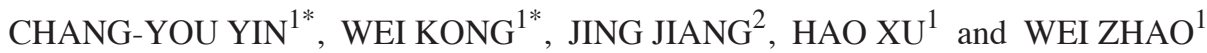 \\ ${ }^{1}$ Department of Neurosurgery, Yantai Yuhuangding Hospital, Yantai, Shandong 264000; \\ ${ }^{2}$ Department of Emergency, Yantaishan Hospital, Yantai, Shandong 264001, P.R. China
}

Received November 30, 2017; Accepted November 16, 2018

DOI: $10.3892 / \mathrm{ol} .2018 .9777$

\begin{abstract}
MicroRNAs (miRNAs/miRs) have been revealed to influence the development and progression of glioblastoma. Although a number of miRNAs are abnormally expressed in glioblastoma it is not clear whether they are a factor associated with glioblastoma pathogenesis. In the present study, miR-7-5p was identified as being aberrantly downregulated in glioblastoma tissues and cell lines. miR-7-5p overexpression significantly decreased the migratory and invasive capacity of the cells, while miR-7-5p silencing had the opposite effect. In addition, a luciferase assay confirmed that special AT rich sequence binding protein 1 (SATB1) was a direct target gene of miR-7-5p in glioblastoma. The overexpression of SATB1 in glioblastoma was revealed to promote cell migration and invasion. In addition, SATB1 overexpression may weaken the inhibitory effect of miR-7-5p on cell migration and invasion. miR-7-5p overexpression reversed the effects of SATB1 on cell migration and invasion in glioblastoma cells. In conclusion, miR-7-5p may be a useful therapeutic target for the diagnosis and treatment of patients with glioblastoma.
\end{abstract}

\section{Introduction}

Glioblastoma is a common primary tumor in the central nervous system, with a 5-year survival rate of below 3\% (1). Glioblastoma is characterised by rapid growth, high invasion, and strong resistance to radiotherapy and chemotherapy (2). Although great progress has been made with the use of modern treatments including surgery, radiotherapy, and chemotherapy, the survival time of patients with glioblastoma remains

Correspondence to: Dr Wei Zhao, Department of Neurosurgery, Yantai Yuhuangding Hospital, 20 Yuding East Road, Yantai, Shandong 264000, P.R. China

E-mail: fvz755359@163.com

*Contributed equally

Key words: glioblastoma, microRNA-7-5p, migration, invasion, special AT rich sequence binding protein 1 short (3). Therefore, it is necessary to identify new effective target genes to suppress the development of glioblastoma.

MicroRNAs (miRNAs/miRs) have been reported to influence the post-transcriptional reaction by binding with the 3'-UTR of the corresponding genes to result in translational suppression or degradation (4). Moreover, increasing miRNAs have been reported to control tumorigenesis, including cell growth, migration, invasion, differentiation and apoptosis (5-7). Additionally, the function of miRNAs is different in various types of cancer, such as lung adenocarcinoma, osteosarcoma and nasopharyngeal carcinoma (8-10). Some miRNAs act as tumor suppressors while others as oncogenes. Many miRNAs have been found to regulate the progression of glioblastoma. For instance, miR-137 inhibited the growth of glioblastoma through suppressing EGFR (11). miR-520c inhibited glioma cell migration and invasion by suppressing TGFBRII (12). miR-595 contributed to cell proliferation in human glioblastoma by regulating SOX7 expression (13). Among them, only a slight effect of miR-7 on glioblastoma has been identified $(14,15)$. In addition, miR-7 with inhibitory effect has been identified in various types of cancer, such as colon cancer (16), pancreatic carcinoma (17), non-small cell lung cancer (18) and thyroid papillary cancer (19). Although the effect of miR-7 has been confirmed repeatedly, the regulatory mechanism of the miR-7/special AT rich sequence binding protein 1 (SATB1) axis has not been previously clarified.

This study focused on the change of miR-7-5p expression in glioblastoma. Moreover, the function of miR-7-5p for the migration and invasion of glioblastoma was determined. We found that miR-7-5p repressed cell mobility and invasiveness through regulation of SATB1 in glioblastoma. These observations may lead to a new approach in the treatment of glioblastoma.

\section{Materials and methods}

Clinical samples and cell culture. Forty-eight glioma specimens and adjacent tissues were taken from the Yantai Yuhuangding Hospital (Yantai, China). The patients received no treatment other than surgery, and all participants provided written informed consent. The tissue was frozen in liquid nitrogen and then stored at $-80^{\circ} \mathrm{C}$ in a refrigerator until use. Human glioblastoma cell lines U87 MG ATCC (male malignant gliomas, ATCC HTB-14), whose origin is unknown, and 
U373 MG ATCC (male malignant gliomas, ATCC HTB-17) as mixed astrocytoma cells were purchased from the ATCC and cultured in DMEM supplemented with $10 \%$ fetal bovine serum (FBS). Normal human astrocytes (NHAs) were purchased from ScienCell Research Laboratories (Carlsbad, CA, USA). Although the U373 and U87 cells have been reported to be contaminated or misidentified (20-22), the use of U373 and U87 cells in this study did not affect the outcomes of this research. This experiment was approved by the Institutional Ethics Committee of Yantai Yuhuangding Hospital.

Cell transfection. The miR-7-5p mimic and inhibitor, SATB1 siRNA were obtained from GenePharma (Shanghai, China) and were transferred into U87 or U373 cells with Lipofectamine 2000 (Thermo Fisher Scientific, Inc., Waltham, MA, USA) according to the manufacturers' protocols.

RNA extraction and RT-qPCR. TRIzol reagent (Invitrogen; Thermo Fisher Scientific, Inc.) was used to extract total RNA. RT-qPCR was carried out through the SYBR PCR Master Mix on an ABI PRISM 7900 Sequence Detection System (Applied Biosystems; Thermo Fisher Scientific, Inc.) to detect the expressions of miR-7-5p and SATB1. U6 or GAPDH served as control for miR-7-5p or SATB1. The following primers were used: miR-7 forward, 5'-AAA ACT GCT GCC AAA ACC AC-3' and reverse, 5'-GCT GCA TTT TAC AGC GAC CAA-3'; SATB1 forward, 5'-CAC AGA GGT GTC TTC CGA AAT CTA-3' and reverse, 5'-AAA GCA AGC CCT GAG TTC TGT TA-3'; GAPDH forward, 5'-CGG AGT CAA CGG ATT TGG TCG TAT-3' and reverse, 5'-AGC CTT CTC CA TGG TGG TGA AGA C-3'; U6 forward, 5'-CTC GCT TCG GCA GCA CAT ATA CT-3' and reverse, 5'-ACG CTT CAC GAA TTT GCG TGTC-3'. Amplification reaction protocol was performed for 35 cycles consisting of $94^{\circ} \mathrm{C}$ for $45 \mathrm{sec}, 95^{\circ} \mathrm{C}$ for $15 \mathrm{sec}, 60^{\circ} \mathrm{C}$ for $1 \mathrm{~min}$. The miR-7-5p or SATB1 levels were analyzed using the $2^{-\Delta \Delta C q}$ method (23).

Dual luciferase assay. The wild-type (wt) 3'-UTR of SATB1 or mutant (mut) 3'-UTR of SATB1 were inserted into the pGL3 promoter vector (Sangon Biotech, Shanghai, China) for luciferase reporter experiments. The vector and miR-7-5p mimic were transfected into cells using Lipofectamine 2000 (Invitrogen; Thermo Fisher Scientific, Inc.), and $24 \mathrm{~h}$ after transfection, Dual-Luciferase Reporter Assay system (Beyotime Institute of Biotechnology, Beijing, China) was applied to measure luciferase activity for $30 \mathrm{~min}$.

Transwell migration and invasion assay. Transwell chambers (Corning Inc., Corning, NY, USA) were applied to evaluate the migratory and invasive ability of glioblastoma cells. Transfected cells $\left(5 \times 10^{4}\right)$ without FBS were placed in the top chamber on the non-coated membrane, and then the lower chamber filled with 20\% FBS to induce transfected cells to migrate or invade through the membrane. The cells were placed in the upper chamber with the coated membrane for the invasion assay, and were incubated for the migration and invasion assay for $48 \mathrm{~h}$. The cells were then stained with crystal violet (Beyotime Institute of Biotechnology) at $37^{\circ} \mathrm{C}$ for $30 \mathrm{~min}$. The cells (magnification, $\mathrm{x} 200$ ) were imaged at random using an inverted microscope (Olympus Corporation, Tokyo, Japan).
Western blot analysis. Protein samples were obtained using RIPA buffer. Proteins were separated with $10 \%$ SDS-PAGE and then incubated with 5\% non-fat milk blocked membranes at room temperature. Next we incubated the PVDF membranes overnight at $4^{\circ} \mathrm{C}$ with anti-SATB1 (dilution 1:1,000; rabbit polyclonal; cat. no. ab70004; Abcam, Cambridge, MA, USA), anti-GAPDH (dilution 1:1,000; mouse monoclonal; cat. no. 60004-1-Ig; ProteinTech, Wuhan, China) and subsequently incubated with goat anti-rabbit IgG H\&L (HRP) (dilution 1:3,000; cat. no. ab6721; Abcam) secondary antibody. The $25 \mu$ protein sample was added in the protein loaded per lane. Protein concentration was calculated using bicinchoninic acid (BCA; Beyotime Institute of Biotechnology, Shanghai, China). Then, protein expression levels were measured by ECL detecting system (Thermo Fisher Scientific, Inc.) using Bio-Rad Image-Lab software (Bio-Rad Laboratories, Inc., Hercules, CA, USA).

Statistical analysis. Statistical analysis was performed with GraphPad Prism 6.0 (GraphPad Software, Inc., La Jolla, CA, USA) and SPSS 17.0 (SPSS, Inc., Chicago, IL, USA). Data are presented as mean \pm standard deviation. Statistical analyses between two groups were performed by Student's t-test. Differences among groups were tested by one-way analysis of variance following by a Tukey's post hoc test. $\mathrm{P}<0.05$ was considered to indicate a statistically significant difference.

\section{Results}

Downregulation of miR-7-5p in human glioblastoma cell lines and tissues. In order to better understand the regulation process of miR-7-5p in glioblastoma pathogenesis, we firstly identified miR-7-5p expression in glioblastoma tissues and cell lines. The observations showed that miR-7-5p expression in glioblastoma tissues was much lower than that of normal tissues (Fig. 1A). The decreased expression of miR-7-5p was also identified in U87 and U373 cell lines (Fig. 1B). All these findings indicated that abnormal expression of miR-7-5p may be related to the progression of glioblastoma.

Cell migration and invasion of glioblastoma are inhibited by $m i R-7-5 p$. To further explore the effect of miR-7-5p in glioblastoma, miR-7-5p mimic or inhibitor was transfected into U87 cells. The efficiency of miR-7-5p expression was detected via RT-qPCR (Fig. 2A). Moreover, the Transwell assay showed that the migration and invasion in cells with miR-7-5p mimics were lower than that of miR-NC group (Fig. 2B). On the contrary, the cells with miR-7-5p inhibitor were higher than that of miR-In-NC for migration and invasion (Fig. 2C). The data revealed that miR-7-5p was a tumor suppressive miRNA for glioblastoma through inhibition of cell migration and invasion.

SATB1 was the direct target of miR-7-5p in glioblastoma. Through TargetScan database (http://www.targetscan. org/vert_71/), SATB1 was identified as one of the target genes of miR-7-5p (Fig. 3A). Moreover, we performed the luciferase reporter assay to verify that miR-7-5p directly targeted SATB1. Luciferase activity in the cells containing miR-7-5p mimics and the wild-type of SATB1 
A

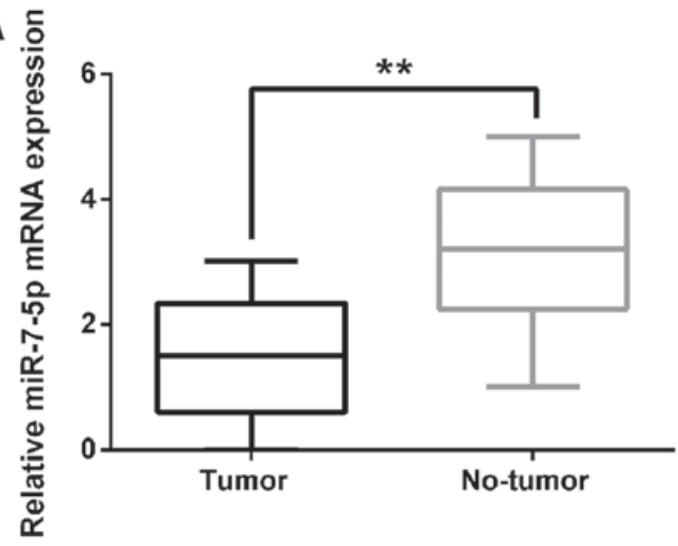

B

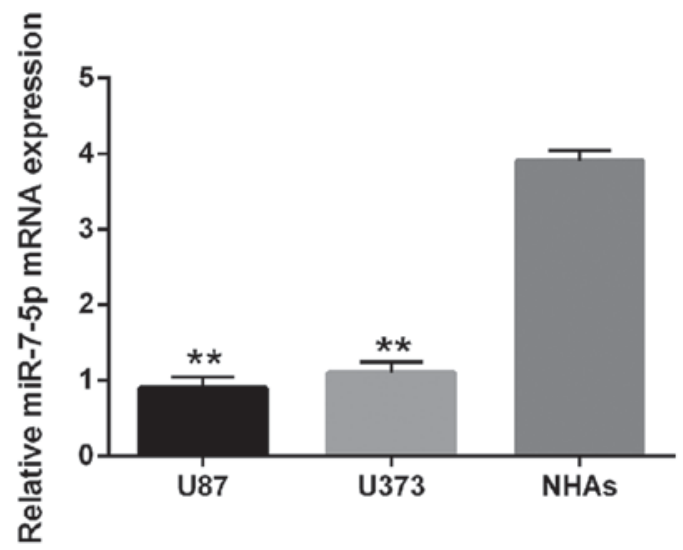

Figure 1. Downregulation of miR-7-5p in human glioblastoma tissues and cell lines. (A) Expression of miR-7-5p in tumor tissues and non-tumor tissues. (B) miR-7-5p expression in U87, U373 and NHAs (control). ${ }^{* *} \mathrm{P}<0.01$. NHAs, normal human astrocytes; miR, microRNA.

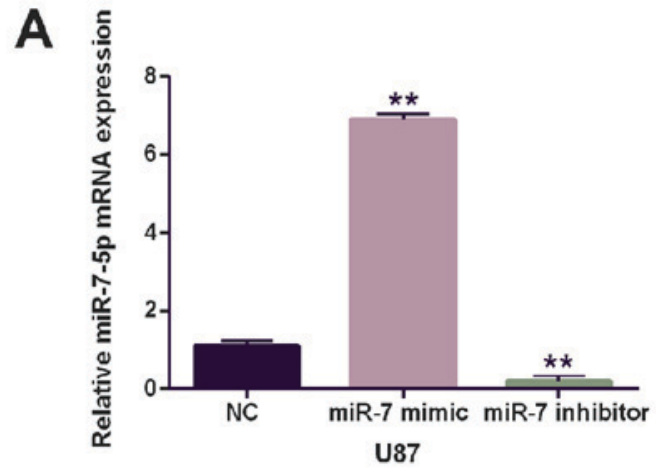

B

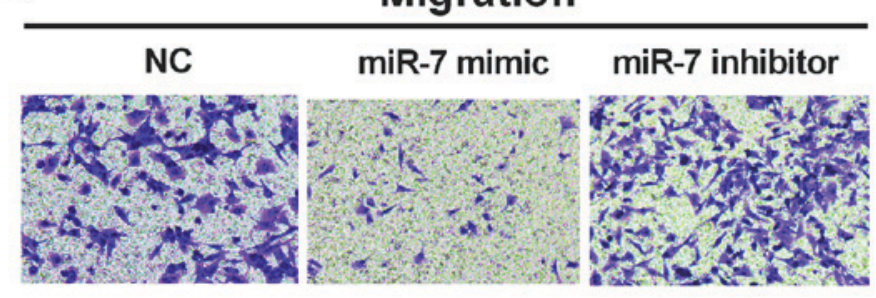

C

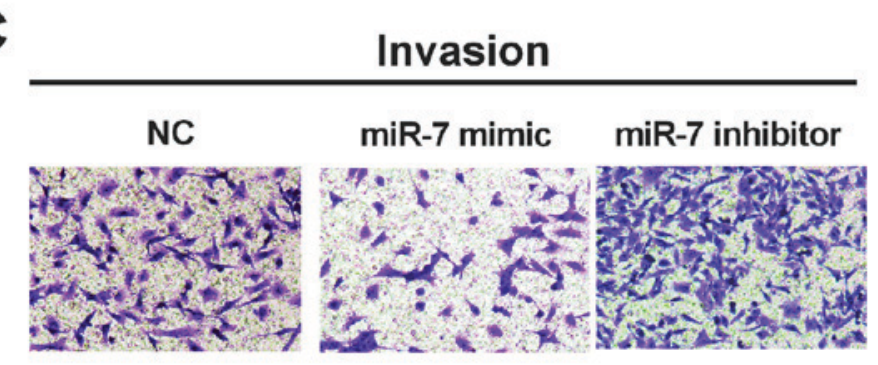

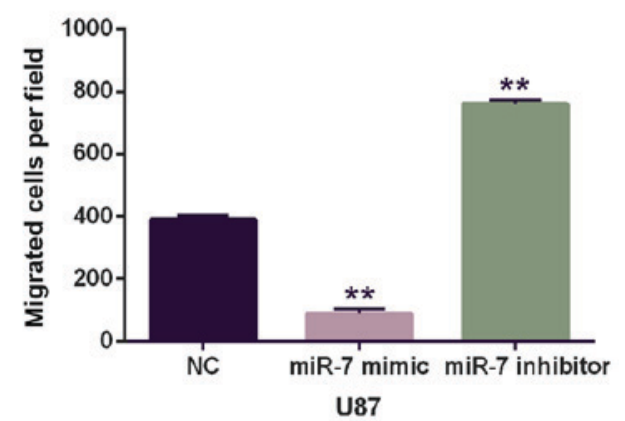

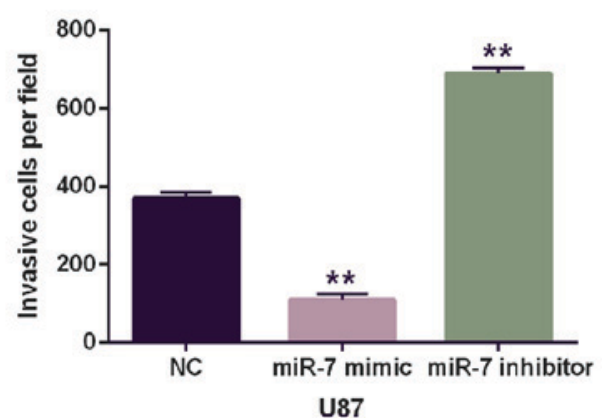

Figure 2. miR-7 inhibits glioblastoma cell migration and invasion. (A) miR-7 mimic or inhibitor was transfected into U87 cells and miR-7 expression was detected via RT-qPCR. (B) Abnormal expression of miR-7 regulated migratory abilities in U87 cells (C) Ectopic expression of miR-7 regulated invasiveness in U87 cells. Magnification, $x 40 .{ }^{* *} \mathrm{P}<0.01$ vs. NC. NC, negative control; miR, microRNA; miR-7, miR-7-5p.

was significantly reduced $(\mathrm{P}<0.01)$ in comparison with the control. Interestingly, there was almost no change in cells containing miR-7-5p mimic and mutant SATB1 (Fig. 3B). According to the luciferase reporter assay, we deduced that
miR-7-5p directly targeted SATB1 in glioblastoma. The SATB1 expression of U87 cells containing miR-7-5p mimics or inhibitor was also detected. Apparently, the mRNA and protein expression levels of SATB1 were significantly 
A

\begin{tabular}{|c|c|}
\hline & $\begin{array}{l}\text { Predicted consequential pairing of target region (top) } \\
\text { and miRNA (bottom) }\end{array}$ \\
\hline $\begin{array}{l}\text { Position 1013-1019 of SATB1 } 3 \text { ' UTR } \\
\text { hsa-miR-7-5p }\end{array}$ & 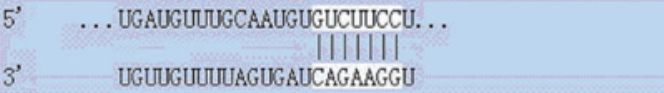 \\
\hline
\end{tabular}

B
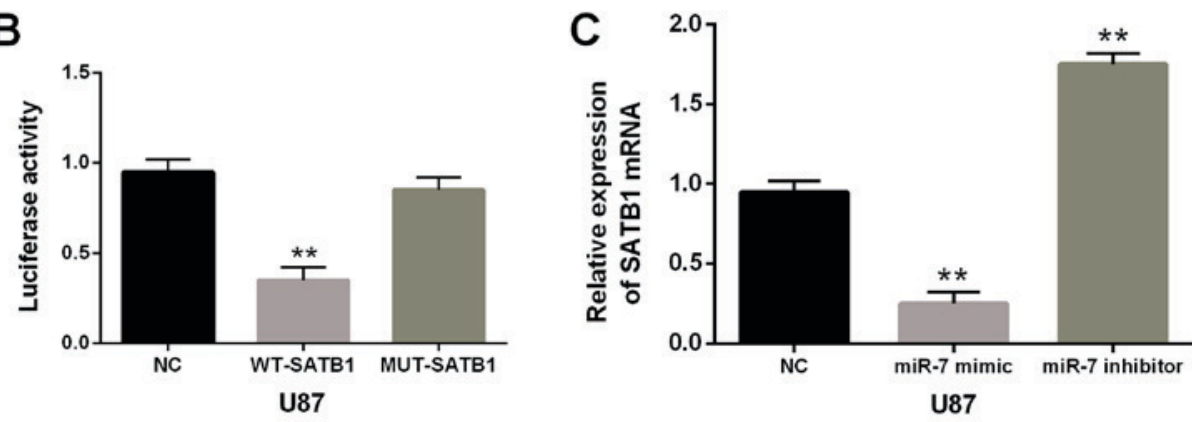

U87

D

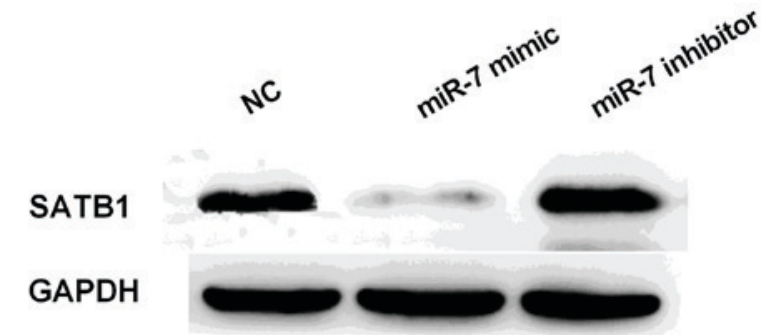

Figure 3. miR-7 directly targets SATB1 in glioblastoma cells. (A) The binding sites of wild-type of SATB1 3'-UTR combined with miR-7-5p. (B) Luciferase reporter assay. The (C) mRNA and (D) protein expression of SATB1 was analyzed in cells containing miR-7 mimic or inhibitor. ${ }^{* *} \mathrm{P}<0.01 \mathrm{vs.} \mathrm{NC.} \mathrm{NC,} \mathrm{negative}$ control; miR, microRNA; SATB1, special AT rich sequence binding protein 1; miR-7, miR-7-5p.

A

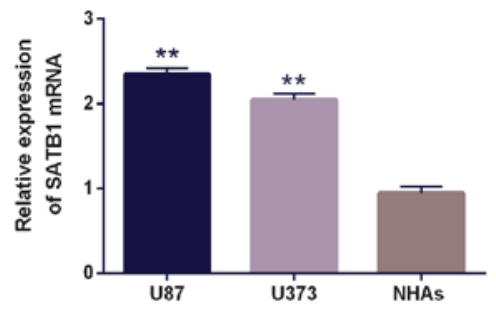

C

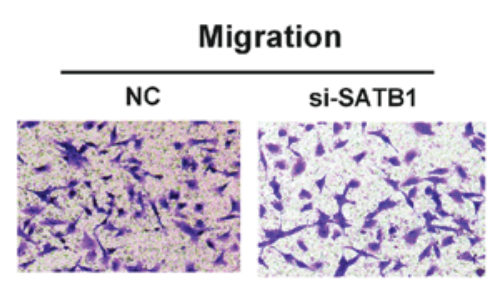

D

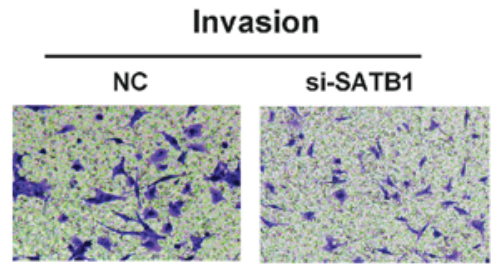

B

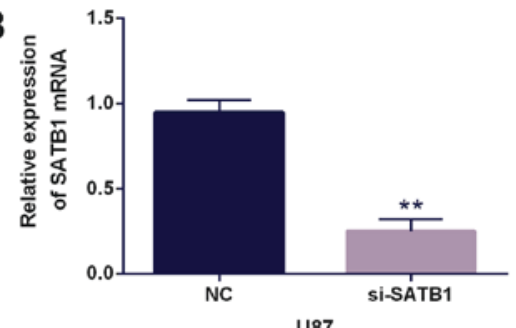

U87
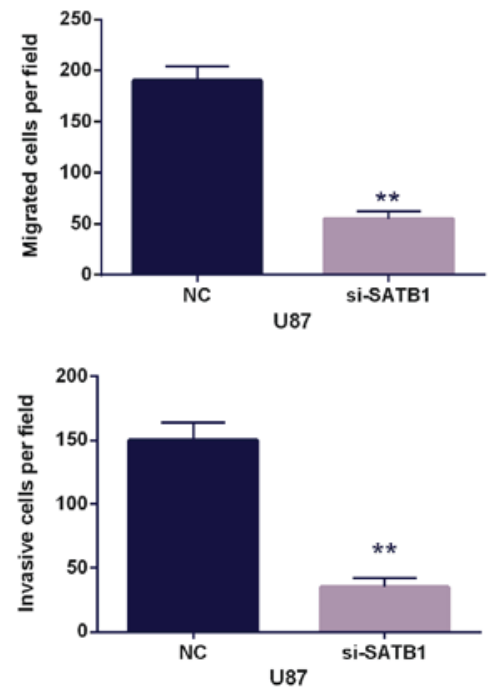

Figure 4. SATB1 promotes glioblastoma. (A) SATB1 expression in glioblastoma as detected by RT-qPCR. (B) SATB1 expression in cells containing si-SATB1 was confirmed by RT-qPCR. (C) Migration and (D) invasion assays in U87 cells containing si-SATB1. Magnification, $\mathrm{x} 40$. ** $<0.01$ vs. NC. NC, negative control; miR, microRNA; SATB1, special AT rich sequence binding protein 1; RT-qPCR, reverse transcription-quantitative polymerase chain reaction; si-, short interfering RNA. 
A

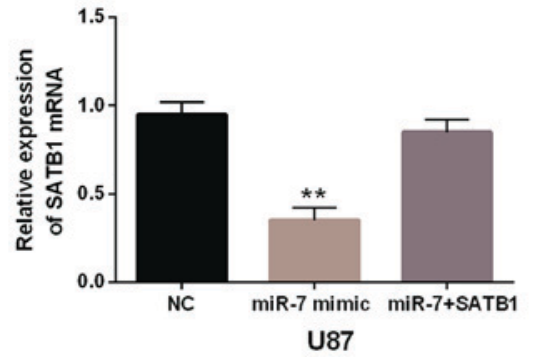

C

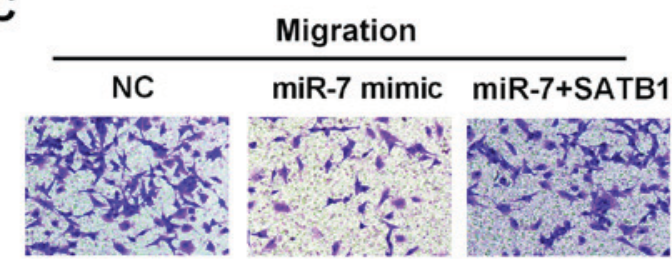

D

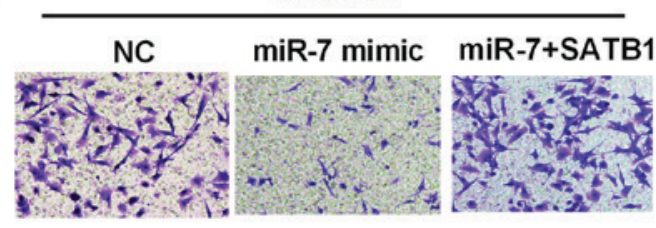

B
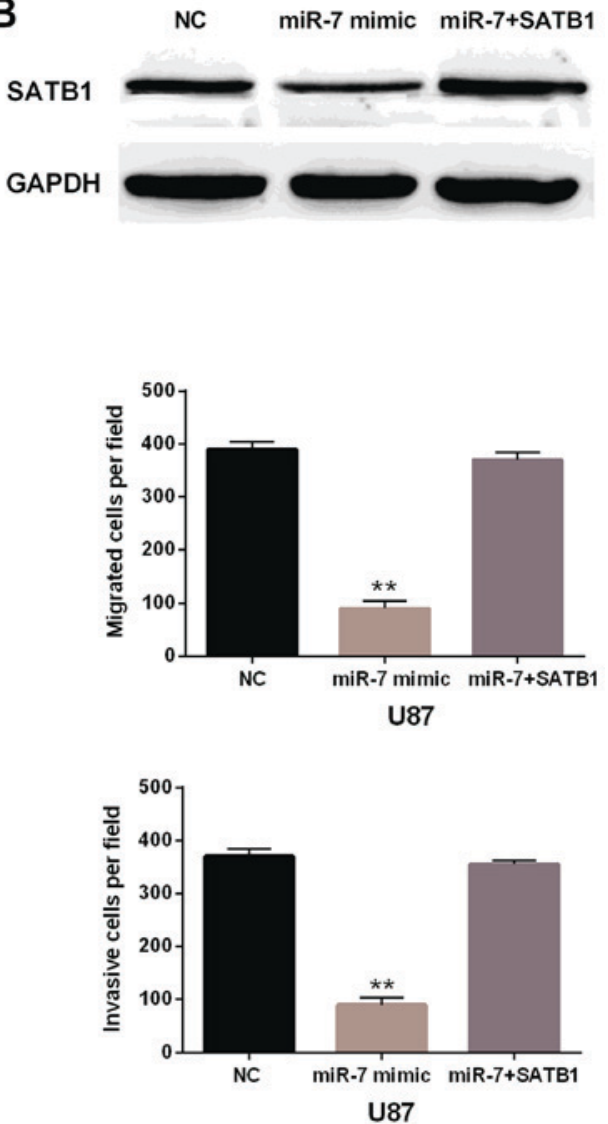

Figure 5. Overexpression of SATB1 inversely reverses the effect of miR-7. (A) Relative mRNA expression of SATB1 in the miR-7 stably-overexpressing cells. (B) The protein expression in cells containing miR-7 and SATB1. (C) Migration and (D) invasion assays were conducted in cells containing miR-7-5p mimic and SATB1. Magnification, $\mathrm{x} 40{ }^{* * *} \mathrm{P}<0.01$ vs. NC. NC, negative control; miR, microRNA; SATB1, special AT rich sequence binding protein 1; miR-7, miR-7-5p.

reduced in U87 cells with miR-7-5p mimics and increased in cells containing miR-7-5p inhibitor in comparison with the control (Fig. 3C and D). In brief, miR-7-5p overexpression inhibited SATB1 expression.

Overexpression of SATB1 inversely reversed the suppressive effect of miR-7-5p in glioblastoma. To further confirm the observation that SATB1 promoted cell migration and invasion in glioblastoma, SATB1 siRNA was used for this investigation. Additionally, the expression of SATB1 was found to be higher in U87 and U373 cell lines than NHA cells (the control, Fig. 4A). SATB1 expression in U87 cells with SATB1 siRNA was decreased vs. the control (Fig. 4B). Migration and invasion were also impaired following SATB1 siRNA expression (Fig. 4C and D). The findings indicated that upregulation of SATB1 in glioblastoma promoted cell migration and invasion in glioblastoma.

Overexpression of SATB1 was speculated to inversely reverse the suppressive effect of miR-7-5p. To confirm that, the negative control or SATB1 expression vector was transfected into U87 cells which the miR-7-5p overexpressed. The mRNA and protein expression levels of SATB1 were decreased in cells with miR-7-5p mimic, whereas little change for SATB1 expression was found in cells with SATB1 plasmid and miR-7-5p mimic compared with the control group (Fig. 5A and B). Importantly, SATB1 overexpression was identified to weaken the inhibitory effect of miR-7-5p on cell migration and invasion in U87 cells (Fig. 5C and D). These results indicated that miR-7-5p suppressed cell migration and invasion in glioblastoma through downregulation of SATB1. The results may indicate the potential to affect tumorigenesis of glioblastoma.

\section{Discussion}

Glioblastoma was reported to develop rapidly and aggressively accounting for $15.4 \%$ of all primary brain tumors and $60-75 \%$ of astrocytoma (24). Although marked progress has been made in understanding the molecular mechanisms underlying the progression of gliomas, no targeted agent has exhibited excellent effects on patient prognosis. Therefore, it is imperative to further understand the pathogenesis of glioblastoma and to develop new targets for effective treatment.

In the present study, it was found that miR-7-5p was downregulated in glioblastoma tissues and cell lines. The overexpression of miR-7-5p inhibited cell migration and invasion of glioblastoma, whereas the downregulation of miR-7-5p had the opposite effect. Moreover, SATB1 was confirmed as a direct target of miR-7-5p. SATB1 was identified to function as an oncogene in glioblastoma. Importantly, the interaction between SATB1 and miR-7-5p was also detected. It indicated that overexpression of SATB1 could impair the suppressive function of miR-7-5p for cell motility in glioblastoma. In brief, miR-7-5p inhibited cell migration 
and invasion of glioblastoma through the suppression of SATB1 expression.

There is increasing evidence that miR-7 makes a contribution to the development of human cancers. Above all, Kefas et al demonstrated that miR-7 was downregulated and inhibited the EGFR and the Akt pathway in glioblastoma (25), and miR-7 interfered with the pathways of PI3K/ATK and Raf/MEK/ERK at the same time to inhibit glioblastoma growth (26). Furthermore, miR-7-5p was found to inhibit vascular endothelial cell proliferation by suppressing RAF1 and be downregulated in glioblastoma microvasculature (27). Regarding cell motility, it was reported that the migration and invasion of glioblastoma cells was inhibited by miR-15b (28), miR-154-5p (29), miR-204 (30) and miR-520c (12) through regulation of IGF1R, PIWIL1, ATF2 and TGFBRII expression. Nevertheless, whether miR-7-5p regulated the cell migration and invasion in glioblastoma was unclear. Our findings indicated that miR-7-5p acted as tumor suppressor in glioblastoma which was similar to previous findings and inhibited the cell migration and invasion as well. Furthermore, miR-7-5p was found to negatively regulate SATB1 expression by binding with its 3'-UTR.

SATB1, a global genome organizer, was reported to be upregulated in renal cell carcinoma (31), osteosarcoma (32) and bladder cancer (33). Han et al demonstrated that SATB1 was involved in the progression and prognosis of glioma (34). Chu et al proved that overexpression of SATB1 was related to the development and progression of glioma (35). Moreover, it was found that SATB1 was repressed by miR-7 in breast cancer (36). In the present study, we found a relationship between miR-7-5p and SATB1 in glioblastoma. Overexpression of SATB1 could weaken the inhibitory effect of miR-7-5p on cell migration and invasion in glioblastoma. Our observation revealed that SATB1 acts as a positive regulator for tumorigenesis of glioblastoma.

In conclusion, results of the current study demonstrated that miR-7-5p was downregulated and overexpression of miR-7-5p inhibited cell migration and invasion in glioblastoma. Moreover, miR-7-5p inhibited cell migration and invasion in glioblastoma by suppressing SATB1 expression.

\section{Acknowledgements}

Not applicable.

\section{Funding}

No funding was received.

\section{Availability of data and materials}

The datasets used and/or analyzed during the present study are available from the corresponding author on reasonable request.

\section{Authors' contributions}

CYY contributed to the study design, data acquisition and analysis and drafted the manuscript. WK contributed significantly to data analysis and manuscript preparation. JJ performed the data analyses. HX assisted in the performance of the analysis with constructive discussions. WZ contributed to the conception of the study. All authors read and approved the final manuscript.

\section{Ethics approval and consent to participate}

The study was approved by the Ethics Committee of Yantai Yuhuangding Hospital (Yantai, China). Signed informed consent was obtained from the patients and/or guardians.

\section{Patient consent for publication}

Not applicable.

\section{Competing interests}

The authors declare that they have no competing interests.

\section{References}

1. Bradshaw A, Wickremsekera A, Tan ST, Peng L, Davis PF and Itinteang T: Cancer stem cell hierarchy in glioblastoma multiforme. Front Surg 3: 21, 2016.

2. Khosla D: Concurrent therapy to enhance radiotherapeutic outcomes in glioblastoma. Ann Transl Med 4: 54, 2016.

3. Franceschi S, Mazzanti CM, Lessi F, Aretini P, Carbone FG, LA Ferla M, Scatena C, Ortenzi V, Vannozzi R, Fanelli G, et al: Investigating molecular alterations to profile short- and long-term recurrence-free survival in patients with primary glioblastoma. Oncol Lett 10: 3599-3606, 2015.

4. Tsai MM, Wang CS, Tsai CY, Huang HW, Chi HC, Lin YH, $\mathrm{Lu} \mathrm{PH}$ and Lin KH: Potential diagnostic, prognostic and therapeutic targets of microRNAs in human gastric cancer. Int J Mol Sci 17: pii: E945, 2016.

5. Dallaire A and Simard MJ: The implication of microRNAs and endo-siRNAs in animal germline and early development. Dev Biol 416: 18-25, 2016.

6. Cimmino A, Calin GA, Fabbri M, Iorio MV, Ferracin M, Shimizu M, Wojcik SE, Aqeilan RI, Zupo S, Dono M, et al: miR-15 and miR-16 induce apoptosis by targeting BCL2. Proc Natl Acad Sci USA 102: 13944-13949, 2005.

7. Asangani IA, Rasheed SA, Nikolova DA, Leupold JH, Colburn NH, Post S and Allgayer H: MicroRNA-21 (miR-21) post-transcriptionally downregulates tumor suppressor Pded4 and stimulates invasion, intravasation and metastasis in colorectal cancer. Oncogene 27: 2128-2136, 2008.

8. Wei S, Zhang ZY, Fu SL, Xie JG, Liu XS, Xu YJ, Zhao JP and Xiong WN: Hsa-miR-623 suppresses tumor progression in human lung adenocarcinoma. Cell Death Dis 8: e2829, 2017.

9. Gui ZL, Wu TL, Zhao GC, Lin ZX and Xu HG: MicroRNA-497 suppress osteosarcoma by targeting MAPK/Erk pathway. Bratisl Lek Listy 118: 449-452, 2017.

10. Wu RS, Qiu EH, Zhu JJ, Wang JR and Lin HL: MiR-101 promotes nasopharyngeal carcinoma cell apoptosis through inhibiting Ras/Raf/MEK/ERK signaling pathway. Eur Rev Med Pharmacol Sci 22: $150-157,2018$

11. Zhang Z, Song X, Tian H, Miao Y, Feng X, Li Y and Wang H: MicroRNA-137 inhibits growth of glioblastoma through EGFR suppression. Am J Transl Res 9: 1492-1499, 2017.

12. Hu S, Chen H, Zhang Y, Wang C, Liu K, Wang $\mathrm{H}$ and Luo J: MicroRNA-520c inhibits glioma cell migration and invasion by the suppression of transforming growth factor- $\beta$ receptor type 2 . Oncol Rep 37: 1691-1697, 2017.

13. Hao Y, Zhang S, Sun S, Zhu J and Xiao Y: miR-595 targeting regulation of SOX7 expression promoted cell proliferation of human glioblastoma. Biomed Pharmacother 80: 121-126, 2016.

14. Babae N, Bourajjaj M, Liu Y, Van Beijnum JR, Cerisoli F, Scaria PV, Verheul M, Van Berkel MP, Pieters EH, Van Haastert RJ, et al: Systemic miRNA-7 delivery inhibits tumor angiogenesis and growth in murine xenograft glioblastoma. Oncotarget 5: 6687-6700, 2014. 
15. Zhang X, Zhang X, Hu S, Zheng M, Zhang J, Zhao J, Zhang X, Yan B, Jia L, Zhao J, et al: Identification of miRNA-7 by genomewide analysis as a critical sensitizer for TRAIL-induced apoptosis in glioblastoma cells. Nucleic Acids Res 45: 5930-5944, 2017.

16. Zeng CY, Zhan YS, Huang J and Chen YX: MicroRNA-7 suppresses human colon cancer invasion and proliferation by targeting the expression of focal adhesion kinase. Mol Med Rep 13: 1297-1303, 2016.

17. Bi Y, Shen W, Min M and Liu Y: MicroRNA-7 functions as a tumor-suppressor gene by regulating ILF2 in pancreatic carcinoma. Int J Mol Med 39: 900-906, 2017.

18. Cao Q, Mao ZD, Shi YJ, Chen Y, Sun Y, Zhang Q, Song L and Peng LP: MicroRNA-7 inhibits cell proliferation, migration and invasion in human non-small cell lung cancer cells by targeting FAK through ERK/MAPK signaling pathway. Oncotarget 7: 77468-77481, 2016.

19. Hua K, Jin J, Zhang $\mathrm{H}$, Zhao B, Wu C, Xu H and Fang L: MicroRNA-7 inhibits proliferation, migration and invasion of thyroid papillary cancer cells via targeting CKS2. Int J Oncol 49: 1531-1540, 2016

20. Grens K: Popular tumor cell line contaminated. The Scientist, Midland,Ontario,2016.https://www.the-scientist.com/? articles. view/ articleNo/46929/title/Popular-Tumor-Cell-LineContaminated/. Updated August 31, 2016.

21. Gasteiger E: ExPASy Bioinformatics Resource Portal (2003) https://web.expasy.org/cellosaurus/CVCL_2219.

22. Allen M, Bjerke M, Edlund H, Nelander S and Westermark B: Origin of the U87MG glioma cell line: Good news and bad news. Sci Transl Med 8: 354re3, 2016.

23. Livak KJ and Schmittgen TD: Analysis of relative gene expression data using real-time quantitative PCR and the 2(-Delta Delta C(T)) method. Methods 25: 402-408, 2001

24. Short SC: Survival from brain tumors in England and Wales up to 2001. Br J Cancer 99 (Suppl 1): S102-S103, 2008.

25. Kefas B, Godlewski J, Comeau L, Li Y, Abounader R, Hawkinson M, Lee J, Fine H, Chiocca EA, Lawler S and Purow B: MicroRNA-7 inhibits the epidermal growth factor receptor and the AKT pathway and is down-regulated in glioblastoma. Cancer Res 68: 3566-3572, 2008

26. Liu Z, Jiang Z, Huang J, Huang S, Li Y, Yu S, Yu S and Liu X: miR-7 inhibits glioblastoma growth by simultaneously interfering with the PI3K/ATK and RAF/MEK/ERK pathways. Int J Oncol 44: 1571-1580, 2014.
27. Liu Z, Liu Y, Li L, Xu Z, Bi B, Wang Y and Li JY: miR-7-5p is frequently downregulated in glioblastoma microvasculature and inhibits vascular endothelial cell proliferation by targeting RAF1. Tumor Biol 35: 10177-10184, 2014.

28. Wang J, Liu H, Tian L, Wang F, Han L, Zhang W and Bai YA miR-15b inhibits the progression of glioblastoma cells through targeting insulin-like growth factor receptor 1 . Horm Cancer 8 : 49-57, 2017.

29. Wang X, Sun S, Tong X, Ma Q, Di H, Fu T, Sun Z, Cai Y, Fan W, Wu Q, et al: miRNA-154-5p inhibits cell proliferation and metastasis by targeting PIWIL1 in glioblastoma. Brain Res 1676: 69-76, 2017

30. Song S, Fajol A, Tu X, Ren B and Shi S: miR-204 suppresses the development and progression of human glioblastoma by targeting ATF2. Oncotarget 7: 70058-70065, 2016

31. Lv C, Bai Z, Liu Z, Luo P and Zhang J: MicroRNA-495 suppresses human renal cell carcinoma malignancy by targeting SATB1. Am J Transl Res 7: 1992-1999, 2015.

32. Wang G, Li B, Fu Y, He M, Wang J, Shen P and Bai L: miR-23a suppresses proliferation of osteosarcoma cells by targeting SATB1. Tumour Biol 36: 4715-4721, 2015.

33. Han B, Luan L, Xu Z and Wu B: Expression and biological roles of SATB1 in human bladder cancer. Tumor Biol 34: 2943-2949, 2013.

34. Han S, Xia J, Qin X, Han S and Wu A: Phosphorylated SATB1 is associated with the progression and prognosis of glioma. Cell Death Dis 4: e901, 2013.

35. Chu SH, Ma YB, Feng DF, Zhang H, Zhu ZA, Li ZQ and Jiang PC: Upregulation of SATB1 is associated with the development and progression of glioma. J Transl Med 10: 149, 2012.

36. Mcinnes N, Sadlon TJ, Brown CY, Pederson S, Beyer M, Schultze JL, McColl S, Goodall GJ and Barry SC: FOXP3 and FOXP3-regulated microRNAs suppress SATB1 in breast cancer cells. Oncogene 31: 1045-1054, 2012.

This work is licensed under a Creative Commons Attribution-NonCommercial-NoDerivatives 4.0 International (CC BY-NC-ND 4.0) License. 